\title{
Chronic prostatic infection: Microbiological findings in two Mediterranean populations
}

\author{
Konstantinos Stamatiou ${ }^{1}$, Vittorio Magri ${ }^{2}$, Gianpaolo Perletti ${ }^{3,4}$, Vaia Papadouli ${ }^{5}$, Nectaria Recleiti ${ }^{5}$, \\ Vassiliki Mamali ${ }^{5}$, Olympia Zarkotou ${ }^{5}$ \\ ${ }^{1}$ Urology Department, Tzaneion Hospital, Piraeus, Greece; \\ ${ }^{2}$ Urology Secondary Care Clinic, ASST-Nord, Milan, Italy; \\ ${ }^{3}$ Department of Biotechnology and Life Sciences, Section of Medical and Surgical Sciences, Università degli Studi dell'Insubria, \\ Varese, Italy; \\ ${ }^{4}$ Faculty of Medicine and Medical Sciences, Ghent University, Ghent, Belgium; \\ ${ }^{5}$ Microbiology Department, Tzaneion Hospital, Piraeus, Greece.
}

\begin{abstract}
Summary Introduction/Aim: Despite accumulated knowledge, several microbiological aspects of chronic bacterial prostatitis (CBP) remain uncertain. The aim of our study was to determine microbiological characteristics on our CBP population.

Materials: The material of this retrospective study consisted in bacterial isolates from urine and/or prostatic secretions or sperm cultures (total ejaculate) obtained from individuals with prostatitis symptoms and from patients with febrile relapses of CBP visiting our department, from 03/2009 to 03/2015.

Retrospective data from an Italian single-center database (years 2009-2015) were also collected for a tentative comparison of pathogen prevalence between chronic bacterial prostatitis cases assessed in Greece and Italy.

Results: A total of 389 bacterial isolates obtained from eligible Greek patients constituted the material of the study. While E coli was the most frequent individual pathogen, Gram-positive species were overly more frequent than Gram-negative. Besides the high frequency of E. coli and E. faecalis isolates the most remarkable similarity between Greek and Italian databases was the wide array of different Gram-positive and Gram-negative species isolated from CBP patients.

Conclusions: In Greece, the incidence of CBP is possibly higher than that reported in international surveys. Similarities between Greek and Italian databases suggest geographical trends in CBP epidemiology.
\end{abstract}

KEY WORDS: Chronic prostatitis; Prostate; Infection; StameyMeyers.

Submitted 19 June 2019; Accepted 21 July 2019

\section{INTRODUCTION/AIM}

Chronic bacterial prostatitis (CBP) is an inflammatory condition of the prostate, characterized by pain in the genital or the pelvic area, which can lead to urinary disorders and may cause sexual dysfunction. Despite the extensive knowledge accumulated over time, several microbiological aspects remain uncertain. The aim of our study was to describe the microbiological characteristics of patients referring to a single Hellenic tertiary care center with symptoms of prostatitis combined with symptoms in the genitourinary tract.

\section{Methods}

\section{Material}

The material of this retrospective study consisted in bacterial isolates from urine and/or prostatic secretions or sperm cultures (total ejaculate) obtained from individuals with reported pelvic discomfort and genital pain, with or without lower urinary tract symptoms and sexual dysfunction, and from patients with febrile relapses of CBP, visiting our department from 03/2009 to 03/2015. Retrospective data from an Italian single-center database (years 2009-2015) were also collected for a tentative comparison of pathogen prevalence's between CBP cases assessed in Greece and Italy.

\section{Patients assessment}

Demographic, microbiological and clinical history of each assessed patient were reviewed. Patients suffering from conditions that influence bacterial virulence or host response (eg. immunodeficiency, abnormalities of the urogenital system) and patients who received antibiotics or immunosuppressive treatment within 4 weeks of the visit were excluded from the study.

Included patients were clinically evaluated and underwent the Meares-Stamey "4-glass" test. Few cases underwent the "two-glass" test, assessing the sole VB2 and VB3 specimens. Depending on medical history and specific symptoms, urethral smear cultures and total ejaculate cultures were additionally obtained from several patients. Patients presenting with febrile prostatitis were investigated by a midstream urine culture (MUC) only. Appropriate antimicrobials were administered to confirmed cases of CBP accordingly to antibiogram for a period of 4 weeks. Follow-up included interview, physical examination and the Meares-Stamey test.

\section{Microbiological evaluation}

The Meares-Stamey test was considered positive when: 1) bacteria grew in the culture of expressed prostatic secretion (EPS) and VB3 urine sample and did not in VB1 and VB2 sample; 2) bacterial colonies in VB3 were higher in num- 
ber compared to VB1 and VB2 samples. Given that no standard cut-off level of the number of bacteria in both urine and prostate secretion samples is defined by consensus for the diagnosis of chronic bacterial prostatitis, we defined no lower acceptable level for either one. Cultures, identification and semi-quantitative assay for Mycoplasma hominis and Ureaplasma urealyticum were performed using the Mycoplasma IST 2 kit (bioMerieux). Chlamydia trachomatis was detected by direct immune-fluorescence (monoclonal antibodies against lipopolysaccharide membrane, Kallestad). Urine samples were cultured undiluted in blood and MacConkey agar plates (Kallestad Lab., TX, USA) and subjected to centrifugation for microscopic examination of the sediment.

Evaluation of culture results was performed by two specialist microbiologists. Identification of traditional pathogens was performed by conventional methods and the Vitek-2 Compact (bioMerieux, France) system and susceptibility testing was performed by disc diffusion and/or the Vitek-2 system. Interpretation of susceptibility results was based on Clinical and Laboratory Standards Institute (CLSI) guidelines.

The local Ethical Committee approved the research protocol for the present retrospective study.

\section{RESULTS}

A total of 548 bacterial isolates were obtained from eligible Greek patients assessed in 1324 visits and recorded during a period of 6 years (2009-2015). In 114 cases the colonies counted in VB2 cultures were as many as those assessed in VB3. In 44 cases the EPS/VB3 cultures were negative despite the presence of bacteria in the specimens. These cases were excluded from the study. Finally, 389 bacterial isolates were recognized as CBP cases and constituted the material of our study. Demographic and microbiological data are presented in Table 1.

\section{Microbiological findings}

A vast variety of pathogens, including rare bacteria, was found in both mono and polymicrobial cultures from both EPS and VB3 samples. The most common were E. coli, coagulase negative staphylococci (CoNS) (mainly S. hominis and S. haemolyticus) and Enterococcus faecalis. The most common combination in polymicrobial isolates composed of two different species was that of E. coli and

Table 1.

Patient demographic and microbiological data.

\begin{tabular}{|lc|}
\hline Clinical sample & Number \\
\hline Number of patients & 389 \\
\hline Median age & 34.7 \\
\hline Microbiological sample & \\
\hline Cultures of prostatic secretions & 92 \\
Urine samples collected after prostate massage & 343 \\
Mid-stream urine only cultures (febrile cases) & 46 \\
Sperm cultures (total ejaculate) & 43 \\
\hline monomicrobial infection & 297 \\
\hline polymicrobial infection & 92 \\
\hline
\end{tabular}

CoNS (21 cases) while in polymicrobial isolates composed of three different species was that of Escherichia coli, Enterococcus faecalis and Proteus mirabilis (4 cases).

29 out of 43 sperm cultures were performed complementary to EPS/VB3. Thirteen of them were identical to EPS/VB3 cultures. The remaining 16 cultures allowed diagnosing bacterial infection while the EPS/VB3 cultures were negative. The most frequently assessed isolates were Enterococcus spp. (19 monomicrobial and 3 polymicrobial). Urethral discharge cultures and cultures from urethral swab samples have diagnosed 48 cases of coexisting urethral infection mainly caused by CoNS and Chlamydia trachomatis (Table 2).

Table 2.

Pathogens found in monomicrobial and polymicrobial isolates.

\begin{tabular}{|lccc|}
\hline Pathogen & EPS/VB3 & Sperm & Urethral \\
\hline Escherichia Coli & 142 & 5 & 1 \\
\hline Coagulase negative staphylococcus & 108 & & 15 \\
\hline Enterococcus faecalis & 102 & 16 & 4 \\
\hline Streptococcus spp. & 31 & & \\
\hline Proteus spp. & 28 & 1 & \\
\hline Staphylococcus aureus & 21 & & 1 \\
\hline Klebsiella spp. & 9 & 5 & \\
\hline Gemella Morbilorum & 4 & 1 & \\
\hline Raoultella planticola & 3 & & \\
\hline Haemophilus parainfluenzae & 3 & 1 & 1 \\
\hline Enterobacter aerogenes & 2 & & \\
\hline Citrobacter freundii & 2 & & \\
\hline Mycoplasma hominis & 1 & 1 & 1 \\
\hline Acinetobacter baumannii & 1 & & \\
\hline Staphylococcus lugdunensis & 1 & \\
\hline Haemophilus influenzae & 1 & \\
\hline Pseudomonas aeruginosa & 1 & \\
\hline Brevundimonas diminuta/vesicularis & 1 & \\
\hline Candida non albicans & 2 & \\
\hline Candida albicans & & \\
\hline Chlamydia trachomatis & & \\
\hline N. gonorrhoeae & & \\
\hline TOTAL & & \\
\hline
\end{tabular}

\section{Follow-up visits and outcome}

As far as the outcomes of follow-up visits are concerned, 263 patients reported elimination of symptoms/clinical improvement, though only 149 were completely cured. Bacterial persistence occurred in 111 cases. In 17 patients pus was found in EPS and/or VB3 in the absence of symptoms. In 6 cases EPS/VB3 cultures were negative despite the presence of bacteria in the same samples. Six patients were diagnosed with another disease during follow-up. In most non-treated cases, the pathogens found in the follow-up cultures were different from those isolated in the initial visit (usually Enterococcus faecalis, CoNS and E. coli). Relapses occurred in 53 patients and almost half of them were caused by microorganisms other than those causing the initial infection. The average time interval between episodes of chronic prostatitis is 13.9 months (minimum 2 and maximum 56 months). The pathogens most commonly associated with clinical relapses were Enterococcus faecalis, CoNS and E. coli. 


\section{Discussion}

\section{Epidemiological and diagnostical issues}

According to the general perception a progression of prostatic inflammation and its consequences from acute to chronic occur resulting on CBP pathogenesis (1). Actually, after an episode of acute bacterial prostatitis approximately $5-10 \%$ of patients progress to chronic infection $(2,3)$.

In accordance to the above, in our study $10.52 \%$ of the patients presented with episodic or persistent relapsing urinary tract infections and all reported a previous diagnosis of prostatitis.

CBP is considered a relatively infrequent disease, since it comprises only $10 \%$ of all prostatitis cases (4).

Nevertheless, from the currently available epidemiologic studies, it appears that CBP is more common since many patients may have bacterial infection despite negative urine cultures: while less than $10 \%$ of men worldwide have a proven bacterial infection of the prostate, up to $25 \%$ receive a diagnosis of prostatitis in their lifetime (5). Negative culture results occur for various reasons including, the presence of fastidious organisms, initiation of antibiotics prior to obtaining an EPS sample, high bacterial count cut-offs established by laboratories (e.g., 50000 CFU), or insufficient sample volumes. Since we defined no lower acceptable level for bacterial colonies in both urine and prostate secretion samples for the diagnosis of chronic bacterial prostatitis, the bacteriologically proven incidence of CBP in this study was $29.45 \%$. Moreover, the fact that we recognised certain Gram-positive bacteria as pathogenic may have also contributed to this difference. In fact, the literature strongly suggests that urologic diseases involving Gram-positive bacteria may be easily overlooked due to limited culture-based assays typically utilized for urine in hospital microbiology laboratories (6).

Insufficient sample volumes explain the low number of assessable EPSs in this study. This fact may indicate the need of better preparing (e.g. abstain from sexual intercourse for 3 to 5 days) before the Meares-Stamey test.

On the other hand, the presence of fastidious organisms, anaerobic pathogens or bacteria not detectable with the usual tests may explain the 44 cases whose EPS/VB3 cultures were negative despite the presence of bacteria. As a matter of fact, traditional culture procedures show lower specificity and sensitivity in detection of bacteria in prostatic specimens than polymerase chain reaction (PCR)-based techniques. Choi et al. found an $11.4 \%$ incidence of bacterial infection in routine EPS or VB3 cultures, while PCR detected bacterial infection in $40.9 \%$ of cases (7).

Similarly, Krieger and Riley found a substantial proportion of positive broadspectrum PCR assays (8). Regardless of its drawbacks, the four-glass test is cur-

Table 3. rently considered the diagnostic standard for $\mathrm{CBP}$, while PCR techniques are cumbersome and have little use in the daily clinical setting. For this reason Magri et al. introduced the 'five-glass' test (four-glass plus post-VB3 semen culture), which showed 3.6- or 6.5-fold increases in relative sensitivity and lesser reductions $(-13.2 \%$ or $14.7 \%$ ) in relative specificity for traditional and unusual pathogens (Mycoplasmata and others) compared with the four-glass or two-glass test, respectively (9). The significance and diagnostic value of complementary semen culture is supported by two other studies (10. 11), though further studies are needed to determine whether a 'five-glass' test may represent a better diagnostic tool. Similarities between Greek and Italian databases may indicate geographical trends in CBP epidemiology.

In fact, similar policies in antibiotic usage and common factors that influence male sexuality and sexual behaviour may contribute to such trends. To our knowledge, a high CBP incidence (26.9\%) has been also demonstrated in a previous Greek study (12) while higher incidences were found in central and southern regions of Italy (13).

\section{Microbiological and pathophysiological issues}

Besides the high frequency of E. coli and E. faecalis isolates the most remarkable similarity between Greek and Italian databases was the wide array of different species like Coag. Neg. Staphylococcus, Streptococcus spp., S. aureus, Haemophylus spp., Citrobacter Spp., Mycoplasma hominis, Haemophylus spp., P. aeruginosa and Candida spp. isolated from both Greek and Italian CBP patients (Tables 2-4).

In our study Enterococcus faecalis was by far the most frequent bacterial isolate from sperm cultures. In the Italian cohort, this species was the third most common isolate in sperm cultures after E. coli and U. urealyticum (Tables

Monomicrobial isolates in an Italian cohort of 151 consecutively assessed Italian patients (years 2009-2015).

\begin{tabular}{|lcccc|}
\hline Pathogen & $\begin{array}{c}\text { Isolated } \\
\text { from EPS/VB3 } \\
\text { only }\end{array}$ & $\begin{array}{c}\text { Isolated } \\
\text { from total } \\
\text { ejaculate only }\end{array}$ & $\begin{array}{c}\text { Isolated } \\
\text { from both } \\
\text { specimens }\end{array}$ & TOTAL \\
\hline Escherichia coli & 26 & 13 & 6 & 45 \\
\hline Proteus mirabilis & 2 & 1 & $/$ & 3 \\
\hline Klebsiella spp. & 1 & 2 & $/$ & 3 \\
\hline Morganella morganii & 1 & 5 & $/$ & 6 \\
\hline Pseudomonas aeruginosa & $/$ & $/$ & 2 & 2 \\
\hline Haemophilus parainfluenzae & $/$ & 2 & $/$ & 2 \\
\hline Citrobacter koseri & $/$ & 1 & $/$ & 1 \\
\hline Neisseria subflava & $/$ & 1 & $/$ & 1 \\
\hline Enterococcus faecalis & 11 & 6 & 3 & 20 \\
\hline Staphylococcus aureus & 3 & $/$ & $/$ & 3 \\
\hline Staphylococcus coagulase-negative & 1 & 5 & 1 & 7 \\
\hline Streptococcus beta-haemolyticus gr. B & $/$ & $/$ & 1 & 1 \\
\hline Streptococcus agalactiae & 1 & $/$ & $/$ & 1 \\
\hline Steptococcus anginosus & $/$ & 1 & $/$ & 1 \\
\hline Kocuria kristinae & $/$ & $/$ & 1 & 1 \\
\hline Chlamydia trachomatis & 3 & $/$ & $/$ & 3 \\
\hline Ureaplasma Urealyticum & 4 & 21 & 2 & 27 \\
\hline Mycoplasma hominis & $/$ & 2 & $/$ & 2 \\
\hline TOTAL & 53 & 60 & 16 & 129 \\
\hline
\end{tabular}


3, 4). Several other studies provided clear evidence of a Gram-positive predomination in bacteriospermia $(14,15)$, while other found a very high proportion of Gram-negative microorganisms with Escherichia coli being the commonest isolate in positive cultures (22.2 to $75 \%$ ) (16-18). Urethral discharge cultures and cultures from urethral swab samples revealed coexisting CoNS and/or Chlamydia trachomatis-induced urethral infection.

Interestingly, in the Italian cohort, whereas Mycoplasmata were found mainly in ejaculate cultures, C. trachomatis was isolated mainly from EPS/VB3 samples (Table 4). Since the urinary tract acts as a nest of infection for the seminal tract, these microorganisms are capable of causing classical infections of the urogenital tract such as epididymitis and prostatitis as well as subclinical reproductive tract infections.
Table 4.

Polymicrobial isolates in an Italian cohort of 151 consecutively assessed Italian patients (years 2009-2015).

\begin{tabular}{|lcccc}
\hline Pathogen & $\begin{array}{c}\text { Isolated } \\
\text { from EPS/VB3 } \\
\text { only }\end{array}$ & $\begin{array}{c}\text { Isolated } \\
\text { from total } \\
\text { ejaculate only }\end{array}$ & $\begin{array}{c}\text { Isolated } \\
\text { from both } \\
\text { specimens }\end{array}$ & TOTAL \\
\hline E.coli + Enterococcus faecalis & 1 & 1 & 2 & 4 \\
\hline E.coli + Klebsiella spp. & $/$ & 1 & $/$ & 1 \\
\hline E.coli + Streptococcus beta-haemolyticus gr. B & 1 & $/$ & $/$ & 1 \\
\hline E.coli + Ureaplasma urealyticum & $/$ & $/$ & 1 & 1 \\
\hline E.coli + Mycoplasma hominis & $/$ & $/$ & 2 & 2 \\
\hline E.coli + Peptostreptococcus spp. & $/$ & $/$ & 1 & 1 \\
\hline E.coli + Candida albicans & $/$ & $/$ & 2 & 2 \\
\hline E. faecalis + Klebsiella spp. & $/$ & 2 & $/$ & 2 \\
\hline E. faecalis + Citrobacter spp. & $/$ & $/$ & 1 & 1 \\
\hline E. faecalis + Ureaplasma urealyticum & $/$ & $/$ & 1 & 1 \\
\hline E. faecalis + Staphylococcus coagulase negative & 1 & $/$ & $/$ & 1 \\
\hline P. aeruginosa + Proteus mirabilis & 1 & $/$ & $/$ & 1 \\
\hline P. aeruginosa + Staphylococcus coagulase negative & $/$ & 1 & $/$ & 1 \\
\hline M. morganii + Haemophilus parainfluenzae & $/$ & 1 & $/$ & 1 \\
\hline Streptococcus mitis + Staphylococcus coagulase negative & $/$ & $/$ & 1 & 1 \\
\hline Chlamydia trachomatis + Ureaplasma urealyticum & $/$ & $/$ & 1 & 1 \\
\hline E. coli + E. faecalis + Staphylococcus coagulase negative & $/$ & $/$ & 1 & 1 \\
\hline TOTAL & 4 & 6 & 12 & 22 \\
\hline
\end{tabular}

\section{Outcome related issues}

Differences in isolated microorganisms in VB3/EPS cultures between follow-up and initial visits of untreated/relapsed cases reinforce the new appreciation of chronic prostatitis as a biofilm disease. Pathogen eradication rates, as high as $80 \%$, have been reported in the pastin CBP patients treated with various fluoroquinolones (19). Our bacterial eradication rate (68.15\%) is similar with that reported in a previous Greek study (64.7\%) (12) and this fact may be attributed to the quinolones overuse in Greece.

\section{Conclusions}

In Greece, the incidence of CBP is possibly higher than that reported in international surveys. Similarities between Greek and Italian databases suggest geographical trends in CBP epidemiology.

The fact that Gram-positive species were the most frequent isolates in both Greek and Italian databases support the role of Gram-positive bacteria in CBPs pathogenicity. Regardless of its drawbacks, the Meares-Stamey is the main tool for the diagnosis of CBP. The significance and diagnostic value of complementary semen culture is supported by our findings, though further studies are needed to determine whether a 'five-glass' test may represent a better diagnostic tool.

\section{REFERENCES}

1. Nickel JC, Olson ME, Barabas A, et al. Pathogenesis of chronic bacterial prostatitis in an animal model. Br J Urol. 1990; 66:47-54.

2. Nickel CJ. Inflammatory and pain conditions of the male genitourinary tract: prostatitis and related pain conditions, orchitis, and epididymitis. Campbell-Walsh Urology. 11 $11^{\text {th }}$ ed. Elsevier; 2016.
3. Yoon BI, Kim S, Han DS, et al. Acute bacterial prostatitis: how to prevent and manage chronic infection? J Infect Chemother. 2012; 18:444-450.

4. Krieger JN, Egan KJ. Comprehensive evaluation and treatment of 75 men referred to chronic prostatitis clinic. Urology. 1991; 38:11-19.

5. Lipsky BA, Byren I, Hoey CT. Treatment of bacterial prostatitis. Clin Infect Dis. 2010; 50:164152.

6. Kline KA, Lewis AL. Gram-positive uropathogens, polymicrobial urinary tract infection, and the emerging microbiota of the urinary tract. Microbiol Spectr. 2016; 4(2).

7. Choi YS, Kim KS, Choi SW, et al. Microbiological etiology of bacterial prostatitis in general hospital and primary care clinic in Korea. Prostate Int. 2013; 1:133-8.

8. Krieger JN, Riley DE. Prostatitis: what is the role of infection. Int J Antimicrob Agents. 2002; 19:475-9.

9. Magri V, Wagenlehner FM, Montanari E, et al. Semen analysis in chronic bacterial prostatitis: diagnostic and therapeutic implications. Asian J Androl. 2009; 11:461-77.

\section{Table 5.}

\section{Clinical and microbiological outcome.}

\begin{tabular}{|lc|}
\hline Cured & 146 \\
\hline Bacterial persistence - Symptom persistence & 63 \\
\hline Bacterial eradication - Symptom persistence & 54 \\
\hline Clinical improvement -Unknown microbiological outcome & 53 \\
\hline Elimination of symptoms - Bacterial persistence & 44 \\
\hline Developed asymptomatic chronic nonbacterial prostatitis & 17 \\
\hline Non-recognizable bacteria in EPS/VB3 cultured samples & 6 \\
\hline Diagnosed with another disease during follow-up & 6 \\
\hline Developed cystitis (VB3 cultures were identical to VB2) & 4 \\
\hline TOTAL & 389 \\
\hline
\end{tabular}


10. Zegarra Montes LZ, Sanchez Mejia AA, Loza Munarriz CA, Gutierrez EC. Semen and urine culture in the diagnosis of chronic bacterial prostatitis. Int Braz J Urol. 2008; 34:30-7.

11. Cai T, Pisano F, Nesi G, et al. Chlamydia trachomatis versus common uropathogens as a cause of chronic bacterial prostatitis: Is there any difference? Results of a prospective parallel-cohort study. Investig Clin Urol. 2017; 58:460-467.

12. Panagopoulos $P$, Antoniadou A, Kanellakopoulou K, et al. Fluoroquinolone treatment of chronic bacterial prostatitis: a prospective cohort study. J Chemother. 2009; 21:317-21.

13. Rizzo M, Marchetti F, Travaglini F, et al. Prevalence, diagnosis and treatment of prostatitis in Italy: a prospective urology outpatient practice study. BJU International. 2003; 92:955-959.

14. Ibadin, OK, Ibeh IN. Bacteriospermia and sperm quality in infertile male patient at University of Benin Teaching Hospital, Benin City, Nigeria. Mal J Microbiol. 2008; 4:65-67.
15. Sheikh AF, Mehdinejad M. Identification and determination of coagulase-negative Staphylococci spp and antimicrobial pattern of isolates from clinical specimens. Afr. J. microbiol. 2012; 6: 16691674

16. Panackal A, Panackal A. Semen Culture a Diagnostic Tool in the Diagnosis of Bacterial Prostatitis. Transl Biomed. 2017; 8:114.

17. Budia A, Luis Palmero J, Broseta E, et al. Value of semen culture in the diagnosis of chronic bacterial prostatitis: a simplified method. Scand J Urol Nephrol. 2006; 40:326-31.

18. Ostrowski A, Banas M, Brzóska Ret al. Semen culture in bacterial prostatitis - retrospective analysis of microbiological profile, antibiograms and clinical utility Eur Urol Suppl 2017; 1:e2949

19. Weidner W, Ludwig M, Brähler E, Schiefer HG. Outcome of antibiotic therapy with ciprofloxacin in chronic bacterial prostatitis. Drugs. 1999; 58 (Suppl 2):103-6.

\section{Correspondence}

Konstantinos Stamatiou, MD

stamatiouk@gmail.com

Urology Dpt, Tzaneion Hospital, Piraeus (Greece)

Vittorio Magri, MD

Urology Secondary Care Clinic, ASST-Nord, Milan, Italy

Gianpaolo Perletti, PhD

Department of Biotechnology and Life Sciences, Section of Medical and Surgical Sciences

Università degli Studi dell'Insubria, Varese (Italy)

Vaia Papadouli, MD

Nectaria Recleiti, MD

Vassiliki Mamali, MD

Olympia Zarkotou, MD

Microbiology Dpt, Tzaneion Hospital, Piraeus (Greece) 